\title{
THE
}

\section{Conformal Invariance, the Central Charge, and Universal Finite- Size Amplitudes at Criticality}

\author{
H. W.J. Blöte \\ John L. Cardy \\ M. P. Nightingale \\ University of Rhode Island, nightingale@uri.edu
}

Follow this and additional works at: https://digitalcommons.uri.edu/phys_facpubs

Terms of Use

All rights reserved under copyright.

\section{Citation/Publisher Attribution}

Blöte, H. W. J., Cardy, J. L., \& Nightingale, M. P. (1986). Conformal invariance, the central charge, and universal finite-size amplitudes at criticality. Physical Review Letters, 56(7), 742-745. doi: 10.1103/ PhysRevLett.56.742

Available at: http://dx.doi.org/10.1103/PhysRevLett.56.742

This Article is brought to you for free and open access by the Physics at DigitalCommons@URI. It has been accepted for inclusion in Physics Faculty Publications by an authorized administrator of DigitalCommons@URI. For more information, please contact digitalcommons-group@uri.edu. 


\title{
Conformal Invariance, the Central Charge, and Universal Finite-Size Amplitudes at Criticality
}

\author{
H. W. J. Blöte \\ Laboratorium voor Technische Natuurkunde, 2600 GA Delft, The Netherlands \\ John L. Cardy \\ Department of Physics, University of California, Santa Barbara, California 93106 \\ and \\ M. P. Nightingale \\ Department of Physics, University of Rhode Island, Kingston, Rhode Island 02881 \\ (Received 21 November 1985)
}

\begin{abstract}
We show that for conformally invariant two-dimensional systems, the amplitude of the finite-size corrections to the free energy of an infinitely long strip of width $L$ at criticality is linearly related to the conformal anomaly number $c$, for various boundary conditions. The result is confirmed by renormalization-group arguments and numerical calculations. It is also related to the magnitude of the Casimir effect in an interacting one-dimensional field theory, and to the low-temperature specific heat in quantum chains.
\end{abstract}

PACS numbers: $64.60 . \mathrm{Fr}, 05.70 . J k, 68.55 .-\mathrm{a}, 75.40 .-\mathrm{s}$

The principle of conformal invariance at a critical point has been shown to be remarkably powerful, especially in two dimensions. ${ }^{1,2}$ Universality classes appear to be characterized by a single dimensionless number $c$, the conformal anomaly or the value of the central charge of the Virasoro algebra. ${ }^{3}$ It was shown by Friedan, Qiu, and Shenker ${ }^{2}$ that unitarity constrains those values of $c$ less than unity to be quantized. For such theories, the critical exponents are given by the $\mathrm{Kac}$ formula, ${ }^{4}$ and the correlation functions are determined. ${ }^{1,5}$ For various models, $c$ has been determined indirectly by use of exact information on exponents and correlation functions obtained by other means. ${ }^{1,2,5}$ In this Letter we give a simple means of determining $c$.

The free energy (measured in units of $k_{\mathrm{B}} T$ ) per unit length of an infinitely long strip of width $L$ at criticality has the finite-size scaling form $F=f L+f^{\times}+\Delta /$ $L+\ldots$, where $f$ is the bulk free energy per unit area, and $\frac{1}{2} f^{\times}$is the surface free energy, which vanishes in the case of periodic boundary conditions. It has been argued, from the assumption that $L^{-1}$ is a scaling field which does not require the introduction of a metric factor, ${ }^{6,7}$ that $\Delta$ is universal. We find that

$$
\Delta=\left\{\begin{array}{l}
-\pi c / 6, \\
-\pi c / 24,
\end{array}\right. \text { periodic boundary conditions, }
$$

free or fixed boundary conditions,

where, in the last case, the order parameter is fixed to the same value on either side of the strip.

These results have several other interesting physical interpretations. Since $F$ corresponds to the groundstate energy of a $(1+1)$-dimensional quantum field theory in a finite volume, Eq. (2) also gives the magnitude of the Casimir effect ${ }^{8}$ in such a theory. The partition function of a classical system of finite width with periodic boundary conditions may also be interpreted as the Feynman path integral for an infinitely long quantum chain at finite temperature $T \propto L^{-1}$. In that case Eq. (1) gives the leading $T \rightarrow 0$ correction to the free energy, from which may be deduced the specific heat $C$. In fact the conformal result applies only if the two-dimensional classical system is rotationally invariant at large distances. This is equivalent to the requirement that the dispersion relation for gapless excitations of the quantum chain is of the form $\omega \sim v k$ with $v=1$. The case $v \neq 1$ can be accommodated by a suitable rescaling of time versus length for the quantum chain. The result is $C \sim \pi c k_{\mathrm{B}}^{2} T / 3 \hbar v$. This is confirmed by exact results for the spin- $\frac{1}{2} X X Z$ chain $^{9}$ $(c=1)$ and for the anisotropic spin- $\frac{1}{2} X Y$ model in a critical transverse field ${ }^{10} \quad\left(c=\frac{1}{2}\right)$. In three dimensions, the analog of $\Delta$ is the interaction energy (in units of $k_{\mathrm{B}} T$ ) per unit area of two plates immersed in a critical system. ${ }^{11}$ Universality in this case was verified by Monte Carlo techniques. ${ }^{12}$ The same constant also plays a role in determining the thickness of gravity-thinned, critical wetting layers. ${ }^{13}$ Two-dimensional analogs of these systems, which would allow an experimental determination of $c$, are conceivable.

A system at a critical point is governed by a reduced fixed-point Hamiltonian ${ }^{14} \mathscr{H}^{*}$. Under a coarse graining in which lengths are rescaled uniformly, the form of the Hamiltonian is invariant. For short-range interactions, the Hamiltonian remains at the fixed point also under conformal transformations, which corre- 
spond to a nonuniform rescaling and rotation. Transformations with a shear component, however, modify the Hamiltonian. The response of $\mathscr{H}$ to such an infinitesimal transformation of the form $x^{\mu}$ $\rightarrow x^{\mu}+\alpha^{\mu}$ is

$$
\delta \mathscr{H}=-\frac{1}{2 \pi} \int \frac{\partial \alpha^{\mu}}{\partial x_{\nu}} T_{\mu \nu} d^{2} x
$$

This defines ${ }^{15}$ the stress tensor $T_{\mu \nu}$. In complex coordinates $(z, \bar{z})$ the only nonzero components are $T_{z z}=T(z)$ and $T_{\overline{z z}}=\bar{T}(\bar{z})$. The conformal anomaly number $c$ may then be defined by ${ }^{1,2}$

$$
\left\langle T(z) T\left(z^{\prime}\right)\right\rangle_{c}=(c / 2)\left(z-z^{\prime}\right)^{-4} \text {. }
$$

Even if $T$ is subtracted so that $\langle T\rangle=0$ in the infinite plane, it is nonzero in the strip. As we now show, its value is related to $\Delta$. Consider the nonconformal transformation $u^{\prime}=u(1-\lambda), \quad v^{\prime}=v(1+\lambda)$, where $\lambda<<1$, and $(u, v)$ measure distances along and across the strip, respectively. According to Eq. (3)

$$
\begin{aligned}
\delta\langle\mathscr{H}\rangle & =-(\lambda / 2 \pi) \int_{-\infty}^{\infty} d u \int_{0}^{L} d v\left\langle-T_{u u}+T_{v v}\right\rangle \\
& =(\lambda L / \pi) \int_{-\infty}^{\infty}(\langle T\rangle+\langle\bar{T}\rangle) d u
\end{aligned}
$$

for the translationally invariant case of periodic boundary conditions. Invariance of the partition function implies that this is compensated by a change in $F$, which is $-2 \lambda \Delta / L$. Hence we find $\Delta=\left(L^{2} / \pi\right)\langle T\rangle$, since $\langle T\rangle=\langle\bar{T}\rangle$ by symmetry. Now the response of $\langle T\rangle$ to $\delta \mathscr{H}$ is

$$
\begin{aligned}
& \delta\langle T(0,0)\rangle \\
& \quad=-(\lambda / \pi) \int_{-\infty}^{\infty} d u \int_{0}^{L} d v\langle T(0,0) T(u, v)\rangle_{c},
\end{aligned}
$$

using $\langle T \bar{T}\rangle_{c}=0$. The connected correlation function $\langle T T\rangle_{c}$ in the strip may be found ${ }^{16}$ by conformal transformation of the infinite-plane result Eq. (4) using the transformation $w=u+i v=(L / 2 \pi) \ln z$. The result is

$$
\langle T(0) T(w)\rangle_{c}=(c / 2)(\pi / L)^{4}[\sinh (\pi w / L)]^{-4}
$$

The integral is divergent as $w \rightarrow 0$, but the final result is independent of the particular method of regularization. The integrals over $u$ and $v$ in Eq. (6) are then elementary, and one obtains $\delta\langle T\rangle=\lambda \pi^{2} c / 3 L^{2}$. This is to be compared with $\delta\langle T\rangle=\delta\left(\pi \Delta / L^{2}\right)$ $=-2 \pi \Delta \lambda / L^{2}$, and the result in Eq. (1) follows.

In the case of free or fixed boundary conditions, the correlation function in the strip is found ${ }^{16}$ with use of the transformation $w=(L / \pi) \ln z$ from the upper half plane. In the latter geometry, the $\langle T T\rangle$ correlation function is as in Eq. (4), while ${ }^{17}\left\langle T\left(z_{1}\right) \bar{T}\left(\bar{z}_{2}\right)\right\rangle$ $=(c / 2)\left(z_{1}-\bar{z}_{2}\right)^{-4}$. However, this term does not contribute to $\Delta$. Thus the only difference between the two cases is that $L$ is replaced by $2 L$. This accounts for the factor of 4 difference between Eqs. (1) and (2).

The results in Eqs. (1) and (2) agree with exact results for the Gaussian model ${ }^{18} \quad(c=1)$ and the Ising model $^{19}\left(c=\frac{1}{2}\right)$. Equation (1) has also been verified $^{20}$ for all the theories in the unitary classification of Friedan, Qiu, and Shenker. ${ }^{2}$ In fact, it is possible to calculate the free energy in an arbitrarily shaped parallelogram with periodic boundary conditions, ${ }^{20}$ of which the infinitely long strip is only a special case.

The result in Eq. (1) can be verified in a modified Gaussian model with reduced Hamiltonian

$$
\mathscr{H}=\frac{1}{2} K^{m} \sum_{k=1}^{m} \sum_{l=1}^{1}\left[\left(\phi_{k, l}-\phi_{k+1, l}\right)^{2}+\left(\phi_{k, l}-\phi_{k, l+1}\right)^{2}\right]+i \alpha \sum_{k=1}^{m} \bar{\sum}^{1}\left(\phi_{k, l}-\phi_{k+1,1}\right),
$$

where the $\phi_{k, l} \in R$ are located at the sites of a simple square lattice on a cylinder, i.e., $\phi_{k, 1}=\phi_{k, L+1}$ for $k=1, \ldots, m$, subject to the constraints $\phi_{1,1}=\phi_{1, s}$ and $\phi_{m, 1}=\phi_{m, s}$ for $s=2, \ldots, L$. The second term in Eq. (7) represents a defect line. A duality transformation changes $K \rightarrow K^{-1}$ in the above Hamiltonian, while the last term becomes $(\alpha / K) \sum_{k=1}^{m-1}\left(\phi_{k, 1}-\phi_{k, L}\right)$. This term may be eliminated by a shift $\phi_{k, l}$ $\rightarrow \phi_{k, l}+\alpha l / L$, which adds a constant $-\alpha^{2}(L-1) /$ $2 K L$ to the free energy per unit length $F$. This modifies $\Delta$ to

$$
\Delta=-\frac{\pi}{6}+\frac{\alpha^{2}}{2 K} .
$$

The defect line is equivalent to charges $\pm \alpha$ at $k=1$ and $k=m$, respectively. As $m \rightarrow \infty$, this is equivalent to a charge $-2 \alpha$ at infinity, as considered by Dotsenko and Fateev. ${ }^{5}$ They found $c=1-24 \alpha^{2}$, when
$K=1 / 8 \pi$, in agreement with Eqs. (1) and (8).

From (8) we derive the value of $\Delta$ for the $q$-state Potts model $(0 \leqslant q \leqslant 4)$ as follows. The critical Potts model can be represented as an $F$ model. ${ }^{21}$ With the usual labeling of the vertices (see Fig. 2 of Ref. 22), the vertex weights are $\left(\omega_{1}, \ldots, \omega_{6}\right)=\left(1,1,1,1, z^{\pi}\right.$ $\left.+z^{-\pi}, z^{\pi}+z^{-\pi}\right)$, where $q^{1 / 2}=2 \cosh \theta$ and $z=e^{\theta / 2}$. As noted in Ref. 21, cylindrical boundary conditions lead to a seam of vertices with modified weights $\omega_{3}^{\prime}=e^{2 \theta}$ and $\omega_{4}^{\prime}=e^{-2 \theta}$. In the body-centered solidon-solid representation of the $F$ model, ${ }^{23}$ the weight of the modified vertices is $e^{2 \theta\left(n_{2}-n_{4}\right)}$, where $n_{2}$ and $n_{4}$ are the column heights at next-nearest-neighbor sites straddling the seam. This corresponds to $\alpha=2 i \theta$ in Eq. (7). The presence of "external" sites ${ }^{21}$ leads to the restriction of constant column height at $k=1, m$, as introduced above. Under renormalization, this body- 
TABLE I. Numerical results for $\Delta$ as a function of the four-spin interaction $K_{4}$ of the Baxter model, obtained from data for $L=4,6, \ldots, 16$. The exact result in this case is $-\pi / 6 \approx-0.523599$.

\begin{tabular}{rlll}
\hline \hline$K_{4}$ & \multicolumn{1}{c}{$\Delta$} & $K_{4}$ & \multicolumn{1}{c}{$\Delta$} \\
\hline-1.0 & -0.525 & 0.1 & -0.523604 \\
-0.8 & -0.524 & 0.2 & -0.523604 \\
-0.6 & -0.524 & 0.3 & -0.523602 \\
-0.4 & -0.5239 & 0.4 & -0.523590 \\
-0.3 & -0.52369 & 0.6 & -0.524 \\
-0.2 & -0.523608 & 0.8 & -0.525 \\
-0.1 & -0.523604 & 1.0 & -0.57 \\
0.0 & -0.523604 & & \\
\hline \hline
\end{tabular}

centered solid-on-solid model flows to the Gaussian model with $22,24,25 K=\pi(2-y)$, where $q^{1 / 2}=2$ $\times \cos (\pi y / 2)$ and $0 \leqslant y \leqslant 2$. Topological objects, such as charges, remain unrenormalized. From Eq. (8) we therefore find

$$
\Delta=-\frac{\pi}{6}+\frac{\pi y^{2}}{2(2-y)} \text {. }
$$

The $q$ dependence of $c$ that we then infer from Eq. (2) agrees with that derived by Kadanoff, and quoted in Ref. 2, and with that conjectured by Dotsenko and Fateev. 5

The same argument can be used for the $O(n)$ model on a hexagonal lattice introduced by Nienhuis, ${ }^{26}$ which can be mapped onto a six-vertex model on a Kagomé lattice, which in turn may be represented by a solidon-solid model. Again a defect line has to be introduced to obtain the correct weights for cylindrical boundary conditions, and the argument proceeds in

TABLE II. Numerical results for $\Delta$ for the $q$-state Potts model compared with exact results derived in the text; freeenergy data of Blöte and Nightingale (Ref. 28) for $L=2, \ldots, 11$.

\begin{tabular}{llc}
\hline \hline$q$ & $\Delta$ & Exact \\
\hline$\frac{1}{64}$ & 0.869148 & 0.869154 \\
$\frac{1}{16}$ & 0.708251 & 0.708256 \\
$\frac{1}{2}$ & 0.233420 & 0.233438 \\
0.95 & 0.0184268 & 0.0184267 \\
1.05 & -0.0176778 & -0.017779 \\
2 & -0.261796 & -0.261799 \\
3 & -0.41892 & -0.41888 \\
4 & -0.52530 & -0.523599 \\
\hline \hline
\end{tabular}

the same way as above. Renormalization maps the $\mathrm{O}(n)$ model onto a Gaussian model with interaction ${ }^{24}$ $K=\pi(2-y)$, where $n=2 \cos (\pi y / 2)$, and $-2 \leqslant y$ $\leqslant 0$. The value of $\Delta$ agrees with Eq. (1) if the $n$ dependence of $c$ conjectured by Dotsenko and Fateev ${ }^{5}$ is used.

The $F$ model and the critical Baxter model ${ }^{27}$ both renormalize onto a Gaussian model with no defect line, and so we expect $\Delta=-\pi / 6$ universally for these cases, in agreement with the idea $^{2}$ that models with continuously varying exponents have $c=1$.

Finally, we present numerical results supporting the expressions derived for $\Delta$ for periodic boundary conditions. The results were obtained from the free energy per site of infinitely long strips of increasing width $L$, by standard extrapolation techniques. ${ }^{28}$ The models in the universality class of the $O(n)$ model that we studied are the continuous $n$-component cubic model defined by Blöte and Nightingale, ${ }^{29}$ in the two cases considered there: $L^{\prime}=0$ and $A=0 \quad\left(e^{-L^{\prime}}=\cosh K\right)$, where $L^{\prime}$ and $K$ are the coefficients of the quadratic and quartic terms in the Hamiltonian.

For the Baxter model in the Ising spin representation $^{30}$ we varied the four-spin and the two equal nextnearest-neighbor interactions $K_{4}$ and $K_{2}$, along the critical line. As shown in Tables I-III, the results agree very well with the theory in all cases, particularly for those values of the parameters where also in previ-

TABLE III. Numerical results for $\Delta$ as a function of $n$ for two special cases ( $L^{\prime}=0$ and $A=0$ ) of the $n$-component cubic model (Ref. 29) extrapolated from data for $L=2, \ldots, 8$, compared with exact results derived in the text.

\begin{tabular}{cccc}
\hline \hline$n$ & $\Delta(A=0)$ & $\Delta\left(L^{\prime}=0\right)$ & Exact \\
\hline-1 & 0.312 & & 0.3142 \\
$-\frac{1}{2}$ & 0.146 & & 0.1461 \\
$-\frac{1}{4}$ & 0.0711 & & 0.0711 \\
$-\frac{1}{8}$ & 0.0352 & & 0.0351 \\
$-\frac{1}{16}$ & 0.0175 & & 0.01746 \\
$-\frac{1}{32}$ & 0.0087 & & 0.00870 \\
$-\frac{1}{64}$ & 0.0044 & & 0.00435 \\
$\frac{1}{64}$ & -0.0043 & -0.00434 & -0.00433 \\
$\frac{1}{32}$ & -0.0087 & -0.00867 & -0.00866 \\
$\frac{1}{16}$ & -0.0173 & -0.00173 & -0.001727 \\
$\frac{1}{8}$ & -0.0344 & -0.0344 & -0.0344 \\
$\frac{1}{4}$ & -0.0682 & -0.0682 & -0.0681 \\
$\frac{1}{2}$ & -0.1343 & -0.134 & -0.1340 \\
1 & -0.262 & -0.262 & -0.2618 \\
2 & -0.523 & -0.525 & -0.5236 \\
\hline \hline
\end{tabular}


ous calculations $s^{6,29,31}$ the asymptotic behavior was observed to set in for those system sizes considered here.

It is a pleasure to acknowledge useful conversations with Jill Bonner, Hubert Knops, Gerhard Müller, Marcel den Nijs, Michael Peskin, and Richard Scalettar. One of us (M.P.N.) thanks the Institut voor Theoretische Fysica of the Katholieke Universiteit Leuven, where part of this work was done, for its hospitality. This research was supported by the U.S. National Science Foundation under Grants No. PHY83-13324 at the University of California at Santa Barbara and No. DMR-8406186 at the University of Rhode Island, by NATO under Grant No. 198/84, and by the Dutch Stichting voor Fundamenteel Onderzoek der Materie.

Note added.-After this paper was submitted for publication, we learned that Affleck $^{32}$ has also obtained the result in Eq. (1).

${ }^{1}$ A. A. Belavin, A. M. Polyakov, and A. B. Zamolodchikov, J. Stat. Phys. 34, 763 (1984), and Nucl. Phys. B241, 333 (1984).

2D. Friedan, Z. Qiu, and S. Shenker, Phys. Rev. Lett. 52, 1575 (1984).

${ }^{3}$ M. A. Virasoro, Phys. Rev. D 1, 2933 (1970).

${ }^{4}$ V. G. Kac, in Group Theoretical Methods in Physics, edited by W. Beiglbock and A. Bohm, Lecture Notes in Physics Vol. 94 (Springer-Verlag, New York, 1979), p. 441.

${ }^{5}$ V1. S. Dotsenko and V. A. Fateev, Nucl. Phys. B240, 312 (1984), and B251, 691 (1985).

${ }^{6}$ M. P. Nightingale and H. W. J. Blöte, J. Phys. A 16, L657 (1983).

${ }^{7}$ V. Privman and M. E. Fisher, Phys. Rev. B 30, 322 (1984).

${ }^{8}$ H. B. G. Casimir, Proc. K. Ned. Akad. Wet. 51, 793 (1948).

${ }^{9}$ M. Takahashi, Prog. Theor. Phys. 50, 1519 (1973), and 51,1348 (1974). For reviews see, e.g., J. C. Bonner, in Magnetostructural Correlations in Exchange-Coupled Systems, edited by R. D. Willett et al. (Reidel, Dordrecht, The Netherlands, 1985).
${ }^{10}$ S. Katsura, Phys. Rev. 127, 1508 (1962).

${ }^{11}$ M. E. Fisher and P.-G. de Gennes, C.R. Acad. Sci. 287, 207 (1978).

${ }^{12}$ K. K. Mon and M. P. Nightingale, Phys. Rev. B 31, 6137 (1985), and unpublished work.

${ }^{13}$ M. P. Nightingale and J. O. Indekeu, Phys. Rev. Lett. 54, 1824 (1985); J. O. Indekeu, M. P. Nightingale, and W. V. Wang, to be published. Here it is exactly verified for the anisotropic Ising model on a triangular lattice that spatial rescaling restores the universality of $\Delta$.

14"Hamiltonian" here means "action" in field-theory language.

${ }^{15}$ The factor of $1 / 2 \pi$ is consistent with the normalization used in Refs. 1 and 2.

16J. L. Cardy, J. Phys. A 17, L385 (1984).

17 J. L. Cardy, Nucl. Phys. B240, 514 (1984).

${ }^{18}$ For the continuum and various lattice versions of the Gaussian model, this calculation can be done exactly. Details will be published elsewhere. The result for free boundary conditions is equivalent to that of $\mathrm{L}$. Brink and $\mathrm{H}$. B. Nielsen, Phys. Lett. 43B, 319 (1973).

${ }^{19}$ A. E. Ferdinand and M. E. Fisher, Phys. Rev. 185, 832 (1969); H. Au-Yang and M. E. Fisher, Phys. Rev. B 11, 3469 (1975).

20 J. L. Cardy, to be published.

${ }^{21}$ R. J. Baxter, S. B. Kelland, and F. Y. Wu, J. Phys. A 9 , 397 (1976).

${ }^{22}$ M. P. M. den Nijs, Phys. Rev. B 27, 1674 (1983).

${ }^{23}$ H. van Beijeren, Phys. Rev. Lett. 38, 993 (1977); H. J. F. Knops, Ann. Phys. (N.Y.) 128, 448 (1981).

${ }^{24}$ B. Nienhuis, J. Stat. Phys. 34, 731 (1984).

${ }^{25}$ The functions $\theta(y)$ and $K(y)$ are multivalued. The correct branch of $\theta(y)$ is chosen by comparison with the Ising case $q=2$.

${ }^{26}$ B. Nienhuis, Phys. Rev. Lett. 49, 1062 (1982).

${ }^{27}$ R. J. Baxter, Ann. Phys. (N.Y.) 70, 193 (1972).

${ }^{28} \mathrm{H}$. W. J. Blöte and M. P. Nightingale, Physica (Amsterdam) 112A, 405 (1982).

${ }^{29} \mathrm{H}$. W. J. Blöte and M. P. Nightingale, Physica (Amsterdam) 129A, 1 (1984).

${ }^{30}$ L. P. Kadanoff and F. Wegner, Phys. Rev. B 4, 3989 (1971); F. Y. Wu, Phys. Rev. B 4, 2312 (1971).

${ }^{31}$ M. P. Nightingale, J. Appl. Phys. 53, 7927 (1982).

32I. Affleck, following Letter [Phys. Rev. Lett. 56, 746 (1986)]. 